\title{
Trends and Issues in Description Logics Frameworks for Image Interpretation
}

\author{
Stamatia Dasiopoulou and Ioannis Kompatsiaris \\ Informatics and Telematics Institute, \\ Centre for Research and Technology Hellas, Thessaloniki, Greece
}

\begin{abstract}
Description Logics have recently attracted significant interest as the underlying formalism for conceptual modelling in the context of high-level image interpretation. Differences in the formulation of image interpretation semantics have resulted in varying configurations with respect to the adopted modelling paradigm, the utilised form of reasoning, and the way imprecision is managed. In this paper, we examine the relevant literature, outlining the corresponding strengths and weaknesses, and argue that although coming up with a complete solution is hard to envisage any time soon, there are a number of key considerations that may serve as guidelines towards this direction.
\end{abstract}

\section{Introduction}

Research in cognitive computer vision is intertwined with the use of symbolic knowledge and reasoning in the pursuit of endowing computational systems with the notion of educated, in terms of background knowledge driven, perception. In the last couple of years, and under the influence of the modelling paradigm embodied in the W3C recommended Semantic Web languages, Description Logics [1] have attracted significant interest as the underlying formalism for conceptual modelling in the context of image interpretation. The low-level information made available by means of typical image analysis is encoded in the form of ABox $A$ assertions, while an appropriately constructed TBox $T$ admits the "reasonable" interpretations that are relevant to the domain of discourse (Fig. 1).

At this point, one would assume that given the standard inference services provided by DLs, the proposed interpretation configurations would differ only with respect to knowledge engineering considerations, such as the type and granularity of the knowledge employed, knowledge acquisition methodologies, and so forth. As a matter of fact though, certain traits that are intrinsic to image interpretation have induced, and in a sense reinforced, the formulation of different interpretation configurations. Ambiguity manifested in the form of incomplete and conflicting assertions $\left(\mathrm{r}_{4}\right.$ in Fig. 1 for example is identified both as building and vegetation by the classification algorithm), constitutes a prominent factor that has induced differing interpretation configurations. Imprecision manifested in the form of degrees of uncertainty (or truth) is another such factor.

Interrelated are the different premises made with respect to the semantics of computational perception per se, which determine how the available knowledge

S. Konstantopoulos et al. (Eds.): SETN 2010, LNAI 6040, pp. 61-70, 2010.

(C) Springer-Verlag Berlin Heidelberg 2010 


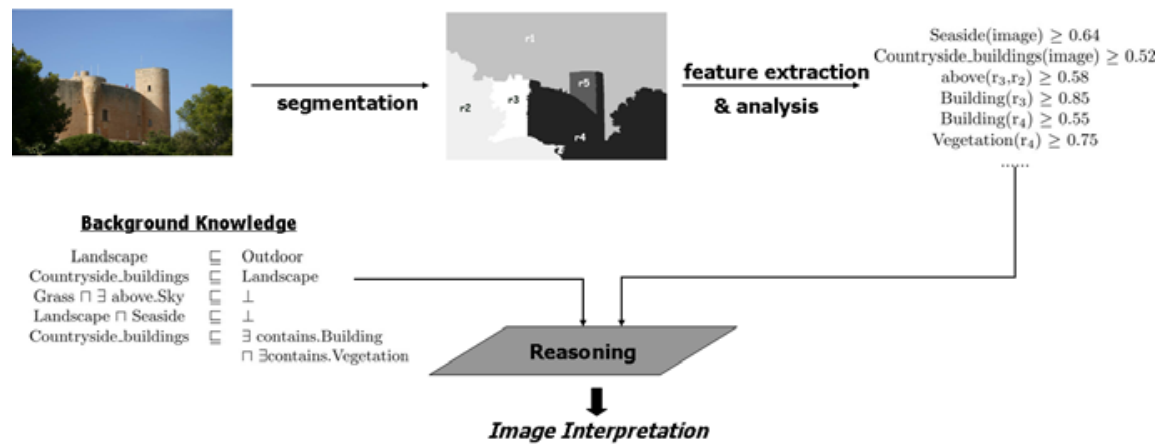

Fig. 1. Abstract architecture of Description Logics image interpretation framework

(axioms and assertions) is to be construed. In effect, the individual viewpoints regarding the transition from low-level representations to high-level semantic interpretations, have a direct impact on which "parts" of the provided semantics are actually deployed and in which ways. As indicated by the undertaken study, it is not uncommon to engage on the closed domain model of the Datalog paradigm rather than the Classical logic paradigm [2].

In this paper, we consider the use of Description Logics in image interpretation and discuss the proposed frameworks with respect to three key conceptual dimensions, namely

- the type of modelling assumption followed, i.e. whether unstated facts are left open

- the form of reasoning followed, and

- the management of imprecision

Section 2 discusses how incompleteness in image interpretation relates to the open world semantics. Section 3 discusses the use of abductive reasoning to better model ambiguity, and Section 4 examines the use of probabilistic and fuzzy extensions for the purpose of handling imprecision. Examining the proposed image interpretation frameworks with respect to these three dimensions, useful considerations emerge with respect to possible directions and guidelines for further research. Section 5 concludes the paper, summarising the main observations.

\section{Open vs. Closed World Semantics}

Description Logics fall under the Classical logics paradigm. The domain is abstractly represented in terms of sets of objects (concepts) and relationships (roles) between them. Appropriate statements (axioms) capture the conditions that need to be met by the "reasonable" states (interpretations) of the domain. There can be many such interpretations, in accordance to all possible ways in 
which objects can be related through the defined relationships in a manner consistent to the defined axioms (open world assumption). Hence, all reasonable interpretations are admitted, but which one is the actual situation is left open. For example, let us assume that Seaside(image), contains(image, region 1 ), contains(image, region ${ }_{2}$ ). In the presence of an axiom stating that seaside images contain at least one region depicting sea, possible interpretations (in the absence of any other information) include that sea is depicted by region ${ }_{1}$, by region ${ }_{2}$, by both, or by some other region, for which we happen to have no available information 1 .

However, in a significant share of the proposed DLs based approaches, image interpretation translates to augmenting the explicitly asserted data, made available via image analysis, with additional ones that are derived through the application of inference over the known only objects and relationships. Indicative approaches include amongst others the works presented in [3|4|5|6|7]. The underlying assumption is that the analysis provided descriptions correspond to all relevant information. Such treatment is closer to the closed world assumption adopted in Datalog related logics, where the only objects and relationships assumed to exist are the explicitly asserted ones, rather than the semantics underlying DLs.

Given the modelling differences between the two paradigms [2], such choice invokes considerations regarding the modelling of image interpretation. In the ideal case, image interpretation would be modelled as mapping a set of meaningfully partitioned regions with well-defined perceptual characteristics to conceptual descriptions, whose further aggregation entails semantic descriptions of higher abstraction. However, the real case is far from the afore-described closed and highly structured setting, which poses hardly pragmatical assumptions with respect to both image processing and analysis, as well as the rendering of semantics in terms of perceptual manifestations. Automatically segmented image regions tend to enclose more than one objects (or constituent parts of multiple objects), as in the case of region $r_{4}$ in Fig. 1 that encloses part of the building and the surrounding vegetation.

As a result, even if accurate and robust perceptual models (in terms of numerical feature values or qualitative representations) are available for the relevant objects and relationships, it is not possible to derive satisfactory interpretations based only on the explicitly asserted regions and their respective descriptions. Similar considerations apply when image analysis provides conceptual descriptions in the form of object and scene for example classifications. Unless all regions correspond to distinct objects (or parts of objects) and all classifications are accurate, the explicitly asserted data comprise an incomplete, partial only view of the actual image content.

The aforementioned example, though representing a small only fraction of the intricacies and challenges that comprise the so called semantic gap [89], outlines

\footnotetext{
${ }^{1}$ Of course, it could be the case that we explicitly admitted interpretations to include exactly two regions, thus ruling out the last possibility. The point made is that the absence of information does not necessarily translate to negative information.
} 
acutely that the kind of incompleteness, that pertains to the open world assumption, percolates image interpretation. Incorporating expectation feedback strategies, where the possible interpretations derived via reasoning serve as cues for subsequent analysis cycles by selectively activating and tuning image processing algorithms, as in [1011], is part of the way towards coping with image interpretation open semantics. Modelling the background knowledge appropriately so as to capture the multiplicity of interpretations due to such incompleteness is another part, essential to the formalisation of image interpretation in a well-defined manner 2 .

A final remark concerns the use of rules. Adopting a hybrid representation scheme, ontologies are used to represent domain and media specific notions, while rules embody the closed domain semantics and provide the mappings between analysis descriptions and semantic ones 312 13. However, the different expressivity capabilities that rules provide, for example the representation of triangular relations as in the case of properties propagating across part-whole relations, seem to have been poorly explored. In the majority of the cases, ontology axioms could have been used as well in the place of rules, signifying that higher familiarity with the rule paradigm may have played a role. Given the aforementioned issues regarding incompleteness, and the continuous efforts towards the formalisation of hybrid representations, a shift towards the effective utlisation of the two formalisms in image interpretation frameworks is to be expected.

\section{Interpretation as Logical Inference}

Standard inference in Description Logics amounts to deductive reasoning. If $\Sigma$ is a logical theory (e.g. background knowledge regarding shape and colour attributes of architectural artifacts) and $\alpha$ a set of facts (e.g. analysis extracted descriptions from images of such artifacts), through deduction is verified whether $\varphi$ (e.g. a building facade) is logically entailed, that is whether $\Sigma, \alpha \models \varphi$.

The majority of the proposed DLs based approaches configure image interpretation along this line of reasoning 14 15 7 16. Whether adopting closed or open world semantics, the higher level interpretations are derived via logical entailment over the available set of analysis provided assertions. The extraction and understanding though of image semantics encompasses a high degree of ambiguity, which as already mentioned in the introductory section, may be manifested in the form of incomplete as well as contradictory information. Ambiguity may as well refer to subjective views and interpretations attributed by different persons, but considerations of this type are beyond the scope of the current discussion.

Unlike the kind of incompleteness, discussed in the previous section, that came from the open world assumption, in this context incomplete information amounts to missing information. Let us consider as an example the opposite case

${ }^{2}$ For example, assuming a region at the top of an image that is asserted as sea, reasoning should be able to derive as a possible interpretation one that construes the upper part of this region as sky and the lower as sea. 
of that described previously, and assume an image that has been partitioned into meaningful, from the conveyed semantics perspective, regions. Although there have been significant advances that support capturing in generic fashion associations between automatically extracted perceptual features and conceptual descriptions, the accuracy of classification remains highly variable and tends to deteriorate rather severely as the number of conceptual notions increases. The situation is further aggravated by serious discrepancies often observed between the intended perceptual to symbolic mappings and the actually acquired ones [17.

As a result, image analysis fails to provide the complete set of expected descriptions (e.g. sky, building), producing instead either false negatives, where the existence of a concept is ignored, or false positives, where it is mistakenly attributed as a different concept. Keeping in mind that meaningful image segmentations are hard to obtain automatically, the aforementioned clearly indicate that adopting a purely deductive form of reasoning, higher level interpretations cannot be derived in a satisfactory and robust manner.

Towards this end, modelling image interpretation as inference to the best explanation using abductive reasoning has been proposed [181920]. Given $\Sigma$ and $\varphi$, abduction consists in finding "explanations" $\alpha$ so that the entailment $\Sigma, \alpha \models \varphi$ is true. The duality between abduction and deduction $(\Sigma, \alpha \models \varphi$ iff $\Sigma, \neg \varphi \mid \neg \alpha$ ) though, is rather misleading regarding the formal apparatus available for abduction [21. Abduction is not mere deduction in reverse 22] and many questions remain still open with respect to the formalisation of minimality criteria and preference metrics driving the generation of explanations. This reflects on the considerably small number of approaches investigating abductive interpretation frameworks, much as on the adoption of kind of ad hoc approaches to the implementation of abductive reasoning.

In 20] for example, Description Logics are used in combination with rules, and abduction is implemented in the form of backward-chaining over the rules. The typical definition for the problem of abduction is modified into $\Sigma, \varphi_{1}, \alpha \models \varphi_{2}$, by splitting $\varphi$ into bona fide assertions $\left(\varphi_{1}\right)$ that are considered true by default, and bona fiats ones $\left(\varphi_{2}\right)$ that need to be explained. This division is arbitrary and in the proposed framework, $\varphi_{2}$ corresponds to the set of spatial relationships assertions. Preference over the possible explanations is determined in terms of the number of (new) individuals that need to be hypothesized (as part of $\alpha$ ) and the number of $\varphi_{2}$ assertions that get explained.

The abductive framework of robot perception presented in 10 is based on a more generic treatment of abduction, but no details are given on the actual computation methodology. Yet, the work presented in [10] and, later, in [19] provide several insights regarding the use of abduction in image interpretation that are not present in 20. Noise and abnormality terms are introduced as part of the background knowledge $\Sigma$, so as to formally account for conflicting

\footnotetext{
${ }^{3}$ Abduction in the context of logic programming is in conflict with the open world semantics underlying the problem of image interpretation, and therefore is not considered here.
} 
assertions as well as for assertions inconsistent with respect to $\Sigma$. The latter is crucial as inconsistencies, due to the implications of the semantics gap, are hardly uncommon in image analysis, and by consequence, in tasks related to interpretation.

Strictly speaking, the presence of inconsistencies introduces already a deviation from the formal abduction problem formulation, as $\Sigma \not \models \neg \varphi$ becomes true. Though the inhibitory implications from inconsistencies affect clearly deductive reasoning as well, it is quite interesting that even in the more straightforward cases of deductive reasoning, the majority of the proposed DLs image interpretation frameworks overlooks this issue. Instead the silent assumption of semantic coherency is made for the analysis provided assertions. Exceptions include [16], where following a reverse like tableau procedure inconsistencies are tracked and resolved, the aforementioned work of [10], and partially 20], as not all of assertions belonging to $\varphi_{2}$ need to be necessarily considered by an interpretation.

\section{Representing and Handling Imprecision}

Besides providing the means to deal with incomplete, missing and contradictory information as discussed in the previous two sections, image interpretation needs to allow for a certain degree of vagueness. Imprecision is present in the extraction of features, the identification of shapes, matching textures, colours, etc., and distills the translation from perceptual to symbolic representations addressed by image analysis. Already in [23, where a first, preliminary proposal of a (crisp) Description Logic language is presented for the recognition of two-dimensional objects, the need for an inference of approximation is highlighted. The wide adoption of statistical models, such as Support Vector Machines (SVMs) [24], Hidden Markov Models [25] and Bayesian Networks (BNs) 26], currently forming the state of the art in image analysis and retrieval frameworks, urges further the investigation of appropriate means to model and handle imprecision in the proposed formal configurations of image interpretation.

Yet, a significant share of the proposed DLs image interpretation frameworks either presume crisp assertions or adopt ad hoc approaches to deal with the degrees of plausibility/vagueness that come with the analysis provided assertions. In 14 27 2011 for example, the assertions over which inference is invoked, are by definition crisp. A pseudo-fuzzy extension is adopted in [4] to allow the definition of conceptual objects in terms of the minimum and maximum accepted values of perceptual features, while in [328] threshold values can be set for individual attributes or features. Clearly though, as membership in perceptual categories is not an all-or-nothing affair, approaches along the aforementioned lines do not capture the pragmatics of image interpretation, and miss significant pieces of information.

In contrast, approaches that deal with imprecision in a more formal and structured fashion embrace either the probabilistic theory [29 or the fuzzy theory 30 . The choice between the two viewpoints reflects the espoused nature of imprecision, and is in accordance with the semantics embodied in the considered image 
analysis techniques. Bayesian and Markov based image analysis frameworks effect probabilistic interpretation, where the degrees accompanying the generated assertions are interpreted as degrees of truth. SVM and analogous approaches deploying similarity-based metrics, translate into degrees of uncertainty.

Concerning probabilistic extensions, related efforts include P-SHOQ $(D)$ [31.32, which is among the most expressive probabilistic description logics that have been investigated, and Pronto [33, a non monotonic probabilistic reasoner built on top of Pellet 34, that however supports only terminological probabilistic knowledge. The lack of correspondence between theoretical advances and corresponding implementations, is reflected in the proposed DLs image interpretation frameworks by exploring notions from the Bayesian networks theory [35/36. Efforts related to this direction include among others PR-OWL 37, which combines first order logic with Bayesian networks, and BayesOWL 38 , which provides a set of rules for the translation of an ontology into an "equivalent" Bayesian network. Again however, although following similar notions, the proposed image interpretation frameworks do not directly use these results.

Image interpretation frameworks that address vagueness on the contrary, exhibit a higher uptake of the corresponding fuzzy extensions to DLs 39|40|4142, an attitude that may be attributed (at least partially) to the availability of respective implementations such as fuzzyDL [43, FiRE [44] and Delorean [45. In 46, fuzzy DLs reasoning is proposed to support the refinement of an initial set of over-segmented image regions and their classifications, in terms of region merging and update of classification degrees based on those of its neighboring regions. In [16], a fuzzy DLs based reasoning framework is proposed to integrate, possibly complementary, overlapping or conflicting classifications at object and scene level, into a semantically coherent final interpretation. A fuzzy spatial relation ontology for image interpretation is presented in [7, yet in the current implementation, only crisp reasoning has been used.

The aforementioned outline, on one hand, the increasing awareness regarding the effective handling of the imprecision involved in image interpretation, and on the other hand, the availability of conducive and active research activities concerning the management of uncertainty and vagueness in Description Logics (for a comprehensive overview the reader is referred to [4]). The different nature of semantics pertaining to the two types of uncertainty [48], and their complementary implications in the context of image interpretation, roughly sketched in the experiments conducted in [10, render the investigation of formal frameworks that couple fuzzy with probabilistic knowledge of particular interest.

\section{Conclusions}

Description Logics have recently gained significant popularity as the underlying formalism for conceptual modelling in formal image interpretation frameworks; a fact not surprising, given the high expressivity and well-defined inference services they come with. Achieving robust and accurate interpretations though, still confronts serious challenges and open issues, in order to bring forth the full potential 
of incorporating knowledge in image interpretation. The open world modelling of the classical logic paradigm on which Description Logics are based, matches closely the incompleteness encountered in image interpretation, urging the investigation of configurations that effectively exploit it, instead of considering only explicitly asserted facts. Conducive towards this end is also the extension of purely deductive reasoning schemes abductive services, which, as outlined appears to be a promising direction for further research. Yet, in order to acquire truly pragmatic interpretation frameworks that model reliably the semantics of both the available facts and the way in which they should be construed, it is mandatory to effectively introduce and handle imprecision in the configured image interpretation models. A promising pursuit for the future is to investigate the coupling of fuzzy and probabilistic reasoning, while preserving clean semantics.

\section{Acknowledgements}

This work was partially supported by the European Commission under contracts FP7-215453 WeKnowIt and FP6-026978 X-Media.

\section{References}

1. Baader, F., Calvanese, D., McGuinness, D.L., Nardi, D., Patel-Schneider, P.F.: The description logic handbook: Theory, implementation, and applications. In: Description Logic Handbook. Cambridge University Press, Cambridge (2003)

2. Patel-Schneider, P., Horrocks, I.: A comparison of two modelling paradigms in the semantic web. J. Web Sem. 5(4), 240-250 (2007)

3. Little, S., Hunter, J.: Rules-by-example - a novel approach to semantic indexing and querying of images. In: McIlraith, S.A., Plexousakis, D., van Harmelen, F. (eds.) ISWC 2004. LNCS, vol. 3298, pp. 534-548. Springer, Heidelberg (2004)

4. Schober, J.P., Hermes, T., Herzog, O.: Content-based image retrieval by ontologybased object recognition. In: KI 2004 Workshop on Applications of Description Logics (ADL), Ulm, Germany september 24, pp. 1-10 (2004)

5. Neumann, B., Moller, R.: On scene interpretation with description logics FBI-B$257 / 04(2004)$

6. Simou, N., Athanasiadis, T., Tzouvaras, V., Kollias, S.: Multimedia reasoning with f-shin. In: 2nd International Workshop on Semantic Media Adaptation and Personalization, London, UK, pp. 413-420 (2007)

7. Hudelot, C., Atif, J., Bloch, I.: Fuzzy spatial relation ontology for image interpretation. Fuzzy Sets and Systems 159(15), 1929-1951 (2008)

8. Smeulders, A.W.M., Worring, M., Santini, S., Gupta, A., Jain, R.: Content-based image retrieval at the end of the early years. IEEE Trans. Pattern Anal. Mach. Intell. 22(12), 1349-1380 (2000)

9. Hanjalic, A., Lienhart, R., Ma, W., Smith, J.: The holy grail of multimedia information retrieval: So close or yet so far away. IEEE Proceedings, Special Issue on Multimedia Information Retrieval 96(4), 541-547 (2008)

10. Shanahan, M.: A logical account of perception incorporating feedback and expectation. In: International Conference on Principles and Knowledge Representation and Reasoning (KR 2002), Toulouse, France, April, 22-25 pp. 3-13 (2002) 
11. Hotz, L., Neumann, B., Terzic, K.: High-level expectations for low-level image processing. In: Dengel, A.R., Berns, K., Breuel, T.M., Bomarius, F., Roth-Berghofer, T.R. (eds.) KI 2008. LNCS (LNAI), vol. 5243, pp. 87-94. Springer, Heidelberg (2008)

12. Dasiopoulou, S., Mezaris, V., Kompatsiaris, I., Papastathis, V., Strintzis, M.: Knowledge-assisted semantic video object detection. IEEE Trans. Circuits Syst. Video Techn. 15(10), 1210-1224 (2005)

13. Bagdanov, A., Bertini, M., DelBimbo, A., Serra, G., Torniai, C.: Semantic annotation and retrieval of video events using multimedia ontologies. In: IEEE International Conference on Semantic Computing (ICSC), Irvine, CA, USA, pp. $713-720(2007)$

14. Moller, R., Neumann, B., Wessel, M.: Towards computer vision with description logics: Some recent progress. In: Workshop on Integration of Speech and Image Understanding, Corfu, Greece, pp. 101-115 (1999)

15. Hunter, J., Drennan, J., Little, S.: Realizing the hydrogen economy through semantic web technologies. IEEE Intelligent Systems Journal - Special Issue on eScience 19, 40-47 (2004)

16. Dasiopoulou, S., Kompatsiaris, I., Strintzis, M.: Applying fuzzy dls in the extraction of image semantics. J. Data Semantics 14, 105-132 (2009)

17. Snoek, C., Huurnink, B., Hollink, L., Rijke, M., Schreiber, G., Worring, M.: Adding semantics to detectors for video retrieval. IEEE Transactions on Multimedia 9(5), 975-986 (2007)

18. Shanahan, M.: Robotics and the common sense informatic situation. In: European Conference on Artificial Intelligence (ECAI), Budapest, Hungary, August, 11-16, pp. 684-688 (1996)

19. Shanahan, M.: Perception as abduction: Turning sensor data into meaningful representation. Cognitive Science 29(1), 103-134 (2005)

20. Espinosa, S., Kaya, A., Melzer, S., Möller, R., Wessel, M.: Multimedia interpretation as abduction. In: International Workshop on Description Logics (DL), BrixenBressanone, Italy, June, 8-10, pp. 323-331 (2007)

21. Elsenbroich, C., Kutz, O., Sattler, U.: A case for abductive reasoning over ontologies. In: Workshop on OWL: Experiences and Directions (OWLED), Athens, Georgia, USA. (November 10-11, 2006)

22. Mayer, M., Pirri, F.: First order abduction via tableau and sequent calculi. Logic Journal of the IGPL 1(1), 99-117 (1993)

23. Sciascio, E.D., Donini, F.: Description logics for image recognition: a preliminary proposal. In: International Workshop on Description Logics (DL), Linköping, Sweden (July 30- August 1, 1999)

24. Burges, C.: A Tutorial on Support Vector Machines for Pattern Recognition. Data Mining and Knowledge Discovery 2(2), 121-167 (1998)

25. Rabiner, L., Juang, B.: An introduction to hidden markov models. ASSP Magazine, IEEE [see also IEEE Signal Processing Magazine] 3(1), 4-16 (1986)

26. Heckerman, D.: A tutorial on learning with bayesian networks. Learning in Graphical Models, 301-354 (1998)

27. Neumann, B., Weiss, T.: Navigating through logic-based scene models for high-level scene interpretations. In: ICVS, pp. 212-222 (2003)

28. Hollink, L., Little, S., Hunter, J.: Evaluating the application of semantic inferencing rules to image annotation. In: International Conference on Knowledge Capture (KCAP), Banff, Alberta, Canada, October 2-5 pp. 91-98 (2005)

29. Nilsson, N.: Probabilistic logic. Artif. Intell. 28(1), 71-87 (1986) 
30. Klir, G., Yuan, B.: Fuzzy sets and fuzzy logic: Theory and applications. PrenticeHall, Englewood Cliffs (1995)

31. Giugno, R., Lukasiewicz, T.: P-shoq(d): A probabilistic extension of shoq(d) for probabilistic ontologies in the semantic web. In: European Conference on Logics in Artificial Intelligence (JELIA), Cosenza, Italy September 23-26, pp. 86-97 (2002)

32. Lukasiewicz, T.: Expressive probabilistic description logics. Artif. Intell. 172(6-7), 852-883 (2008)

33. Klinov, P.: Pronto: A non-monotonic probabilistic description logic reasoner. In: Bechhofer, S., Hauswirth, M., Hoffmann, J., Koubarakis, M. (eds.) ESWC 2008. LNCS, vol. 5021, pp. 822-826. Springer, Heidelberg (2008)

34. Sirin, E., Parsia, B., Grau, B., Kalyanpur, A., Katz, Y.: Pellet: A practical owl-dl reasoner. J. Web Sem. 5(2), 51-53 (2007)

35. Town, C., Sinclair, D.: A self-referential perceptual inference framework for video interpretation. In: International Confernce on Computer Vision Systems (ICVS), Graz, Austria, pp. 54-67 (2003)

36. Neumann, B., Möller, R.: On scene interpretation with description logics. Image Vision Comput. 26(1), 82-101 (2008)

37. da Costa, P., Laskey, K., Laskey, K.: Pr-owl: A bayesian ontology language for the semantic web. In: URSW. LNCS, pp. 88-107 (2008)

38. Ding, Z.: BayesOWL: A Probabilistic Framework for Semantic Web. Phd thesis, University of Maryland, Baltimore County (December 2005)

39. Yen, J.: Generalizing term subsumption languages to fuzzy logic. In: 12th International Joint Conference on Artificial Intelligence (IJCAI), Sydney, Australia, August 24-30, pp. 472-477 (1991)

40. Straccia, U.: Reasoning within fuzzy description logics. J. Artif. Intell. Res (JAIR) 14, 137-166 (2001)

41. Straccia, U.: Transforming fuzzy description logics into classical description logics. In: European Conference on Logics in Artificial Intelligence (JELIA), Lisbon, Portugal, September, 27-30 pp. 385-399 (2004)

42. Stoilos, G., Stamou, G., Tzouvaras, V., Pan, J., Horrocks, I.: The fuzzy description logic f-SHIN. In: International Workshop on Uncertainty Reasoning For the Semantic Web (URSW), Galway, Ireland November, 7 pp. 67-76 (2005)

43. Bobillo, F., Straccia, U.: fuzzydl: An expressive fuzzy description logic reasoner. In: International Conference on Fuzzy Systems (FUZZ) June, 1-6 pp. 923-930. IEEE Computer Society, Hong Kong (2008)

44. Simou, N., Kollias, S.: Fire: A fuzzy reasoning engine for impecise knowledge, In: K-Space PhD Students Workshop Berlin, Germany, September 14 (2007)

45. Bobillo, F., Delgado, M., Gómez-Romero, J.: Delorean: A reasoner for fuzzy owl 1.1. In: International Workshop on Uncertainty Reasoning for the Semantic Web (URSW), Karlsruhe, Germany, October 26 (2008)

46. Simou, N., Athanasiadis, T., Stoilos, G., Kollias, S.: Image indexing and retrieval using expressive fuzzy description logics. Signal, Image and Video Processing 2(4), 321-335 (2008)

47. Straccia, U.: Managing uncertainty and vagueness in description logics, logic programs and description logic programs. In: Tutorial Lectures, Reasoning Web, 4th International Summer School, Venice, Italy, pp. 54-103 (2008)

48. Dubois, D., Prade, H.: Possibility theory, probability theory and multiple-valued logics: A clarification. Annals of Mathematics and Artificail Intelligence 32(1-4), 35-66 (2001) 